\title{
8-years experience with ataxia telangiectasia: A series of 7 cases
}

\author{
Nicole Villagaray-Pacheco ${ }^{1,2}$, Kelly Franco-Bustamante ${ }^{3}$, Wilmer Córdova-Calderón ${ }^{4}$ \\ ${ }^{1}$ Faculty of Medicine, Universidad Nacional Mayor de San Marcos, Lima, Perú \\ ${ }^{2}$ Sociedad Científica de San Fernando, Lima, Perú \\ ${ }^{3}$ Instituto Nacional de Salud del Niño San Borja, Lima, Perú \\ ${ }^{4}$ Instituto Nacional de Salud del Niño, Lima, Perú
}

\begin{abstract}
Introduction. Ataxia telangiectasia (AT) is an autosomal recessive multisystemic neurodegenerative disease, considered a rare and orphan disease.

Methods. A cohort of case series from 2012 to 2020. We included patients younger than 18 years, admitted with diagnosis of ataxia telangiectasia from a tertiary care hospital and national referral center in Peru. Year of diagnostic, mortality, demographics, genetic test, and other main clinical outcomes were described.

Results. A series of seven cases are presented (4 are women, between 6 and 15 years old) from different provinces of Peru. Five cases had a diagnostic delay. Two cases died at 9 and 12 years. Three cases have a brother with AT. The most frequent reason for consultation (6/7) was recurrent lung infection, followed by postural instability. All had delayed psychomotor development, recurrent respiratory, and digestive infections. The main laboratory results were lymphopenia (5 cases), neutropenia (6 cases), IgA deficiency (5 cases), and elevated alpha-fetoprotein (4 cases).

Conclusion. AT considerably deteriorates the quality and prognosis of life of patients.
\end{abstract}

Keywords: ataxia telangiectasia, primary immunodeficiency, Louis-Bar syndrome, rare disease, orphan disease, Peru

\section{INTRODUCTION}

Ataxia telangiectasia (AT) or Louis-Bar syndrome is a multisystemic autosomal recessive genetic disease; due to mutations in the ATM gene located on the long arm of chromosome $11(1,2)$. AT is characterized by progressive cerebellar neurodegeneration, ocular-cutaneous telangiectasias, inmunodeficiencies, recurrent infections, lymphopenia, neutropenia, elevated alphafetoprotein, and insulin resistance. In addition, they present radiosensitivity and susceptibility to develop neoplasms, in particular leukemia and lymphoma (1-4).

The reported global prevalence is between 1 and 2.5 per 100,000 live births (1). AT deteriorates the quality of life of patients and the prognosis for life is unfavorable (5-7).
In Peru, there are few studies on AT with only one case reported (8). Thus, the results of this study will contribute to the knowledge of the clinical and laboratory practice of AT. Therefore, we report this series of cases from a tertiary care hospital and national referral center in Peru.

\section{METHODS}

A cohort of case series of AT. We included patients younger than 18 years, admitted with clinical diagnosis of ataxia telangiectasia at Institute of Child Health in Lima, Peru, between 2012 and 2020. Age of diagnostic delay, mortality, demographics, genetic test, and other main clinical outcomes were described. 
The presence of ATM mutations was analyzed in two probands' DNA (case N 1 y N 3) by next generation sequencing (Bone Marrow Failure Gene Sequencing Panel, Molecular Genetics Laboratory at Instituto Nacional de Salud del Niño San Borja).

Two investigators independently extracted data from each subject who met the inclusion criteria and if there were any differences it was resolved by a third investigator. Subsequently, the data of the subjects was coded, assigning a number to each participant, ensuring the complete confidentiality of the data and information. Manipulation of the subject data was performed solely by the principal investigators.

The research was approved by the Ethics Research Committee of the Faculty of Medicine of the Universidad Nacional Mayor de San Marcos. The authors declare that the procedures followed are in accordance with the World Medical Association and the Declaration of Helsinki.

Demographic and clinical characteristics and auxiliary tests were specified for each case. The qualitative variables were represented by absolute frequencies.

\section{RESULTS}

A series of 7 cases ( 4 women), aged 6 to 15 years, from 2012 to 2020 from a national referral hospital in Peru is presented. Three come from
Junín, the rest from Lima, Chiclayo, Huánuco and Cuzco.

Five cases were diagnosed after 3 years of started ataxia or telangiectasia. Two cases died at 9 and 12 years. Three cases have a brother with AT. The most frequent reason for consultation was recurrent lung infection $(6 / 7,85.7 \%)$. Other reasons were the progressive increase in the support base and postural instability (Table 1).

All had delayed psychomotor development and recurrent respiratory and digestive infections. One case presented reactivation of miliary tuberculosis, another case presented a hamartoma and epilepsy. Three patients have a brother with AT and no case reports consanguinity between parents. In three cases, the mothers developed a urinary tract infection during the 1st trimester of pregnancy (Table 1).

All presented ataxic gait, axial instability, lateralization of the head, dysmetria, alteration of the index-nose maneuver, dysdiadochokinesia, dysarthria, and ocular telangiectasias (Figure 1).

Only one case had telangiectasia in the skin. Two cases presented horizontal nystagmus. The motor system was evaluated where all presented muscular hypotonia, muscular hypotrophy, diminished muscular force and diminished osteotendinous reflexes. Only one case was evaluated with the Romberg test with a negative result (Table 2).

The earliest clinical characteristic was ataxia in 6 cases and ocular telangiectasia in one case. The age of diagnosis of AT in one case was 7 years; in

TABLE 1. Demographic characteristics and personal history

\begin{tabular}{|c|c|c|c|c|c|c|c|}
\hline & Case 1 & Case 2 & Case 3 & Case 4 & Case 5 & Case 6 & Case 7 \\
\hline Sex & Male & Male & Female & Female & Male & Female & Female \\
\hline Age & 15 & $12(+)$ & 10 & 8 & 6 & $9(+)$ & 8 \\
\hline Reason for consultation & $\begin{array}{c}\text { Respiratory } \\
\text { infection }\end{array}$ & $\begin{array}{c}\text { Progressive } \\
\text { increase in the } \\
\text { base of support } \\
\text { and postural } \\
\text { instability }\end{array}$ & $\begin{array}{l}\text { Respiratory } \\
\text { infection }\end{array}$ & $\begin{array}{l}\text { Respiratory } \\
\text { infection }\end{array}$ & $\begin{array}{c}\text { Respiratory } \\
\text { infection }\end{array}$ & $\begin{array}{l}\text { Recurrent } \\
\text { respiratory } \\
\text { infections }\end{array}$ & $\begin{array}{l}\text { Recurrent } \\
\text { respiratory } \\
\text { infections }\end{array}$ \\
\hline Age of diagnosis & 5 & 5 & 5 & 2 & 2 & 6 & 7 \\
\hline Onset of ataxia & 1 yo & 2 yo & 5 yo & 2 yo & 2 yo & 2 yo & 1 yo \\
\hline Onset of telangiectasia & 3 yo & 3 yo & 3 yo & 3 yo & 3 yo & 3 yo & 3 yo \\
\hline \multicolumn{8}{|l|}{ Personal history } \\
\hline Miliary tuberculosis & No & Yes & No & No & No & No & No \\
\hline Siblings with AT & Yes & No & No & No & Yes & Yes & No \\
\hline $\begin{array}{l}\text { Consanguinity between } \\
\text { parents }\end{array}$ & No & No & No & No & No & No & No \\
\hline $\begin{array}{l}\text { Pathology during } \\
\text { pregnancy }\end{array}$ & No & Yes & No & No & Yes & Yes & No \\
\hline
\end{tabular}




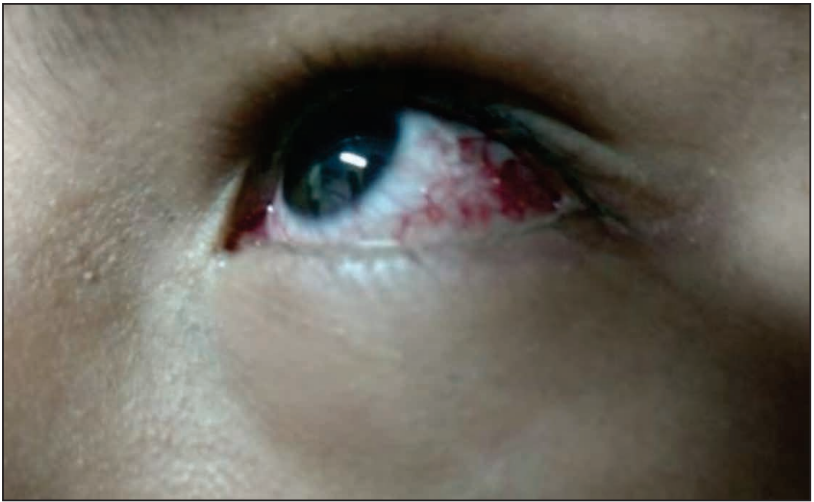

FIGURE 1. Ocular telangiectasias

TABLE 2. Clinical characteristics and auxiliary exams

\begin{tabular}{|c|c|c|}
\hline & & $\begin{array}{l}\text { Cases } \\
\text { N (\%) }\end{array}$ \\
\hline \multicolumn{3}{|c|}{ Clinical characteristics } \\
\hline & Ataxic gait & $7(100 \%)$ \\
\hline & Axial instability & $7(100 \%)$ \\
\hline & Lateralization of the head & $7(100 \%)$ \\
\hline & Dysmetria-dysdiadochokinesia & $7(100 \%)$ \\
\hline & Altered index-nose maneuver & $6(86 \%)$ \\
\hline & Horizontal nystagmus & $2(29 \%)$ \\
\hline & Ocular telangiectasia (scleras) & $7(100 \%)$ \\
\hline & Telangiectasia in skin & $1(14 \%)$ \\
\hline & OTR decreased & $7(100 \%)$ \\
\hline \multicolumn{3}{|l|}{ Laboratory } \\
\hline & Lymphocytes decreased & $5(71 \%)$ \\
\hline & Neutrophils decreased & $6(86 \%)$ \\
\hline & IgA absent & $4(57 \%)$ \\
\hline & IgA normal & $2(29 \%)$ \\
\hline & IgA decreased & $1(14 \%)$ \\
\hline & IgG normal & $6(86 \%)$ \\
\hline & IgG decreased & $1(14 \%)$ \\
\hline & IgM normal & $6(86 \%)$ \\
\hline & IgM elevated & $1(14 \%)$ \\
\hline & IgE normal & $6(86 \%)$ \\
\hline & IgE decreced & $1(14 \%)$ \\
\hline & AFP & $7(100 \%)$ \\
\hline Chest x-ray & & $5(14 \%)$ \\
\hline
\end{tabular}

another case, at 6 years; three cases, at 5 years and in two cases, at 2 years (Table 2).

The laboratory results were the presence of lymphocytopenia, in 5 cases; neutropenia, in 6 cases; IgA was absent in 4 cases and decreased in 1 case; IgG was decreased in 1 case; IgM was increased in 1 case; only 4 cases had alpha-fetoprotein measured, presenting elevation; only 2 cases were tested for insulin resistance without resistance; hemoglobin was decreased in 2 cases. Five cases underwent radiation for recurrent respiratory infections.
Case 1 was found to carry the compound heterozygosity mutation

ATM: c.5650_5657delACAACCCC (NM_000051.3), c.38G $>$ A (NM_000051.3) and c.2376+2T $>$ G (NM_000051.3). The first variant of uncertain significance (VUS) revealed Thr1884Cy sfsTer17, which ATM protein was resulting in a truncated product, the second VUS caused splice site mutation and third VUS caused Arg13His. Case 3 was found to carry to carry the compound heterozygosity mutation ATM: c.2466+5G $>$ C (NM_000051.3) and c.2838+4A $>\mathrm{G}$ (NM_000051.3). This variants of uncertain significance caused splice site mutations.

There is no cure for AT, only palliative treatment to improve quality of life. 5 patients are given an intravenous infusion of Human Immunoglobulin 10\% monthly. All patients received physical stimulation therapy and respiratory therapy, antibiotics for recurrent respiratory infections, bronchodilators and, only in the case of epilepsy, anticonvulsants.

The patients have received a multidisciplinary follow-up through immunology consultations, in addition to monitoring for the risk of neoplasia; pulmonology for the control of recurrent respiratory infections; to neurology for the follow-up and supervision of the progress of the manifestations of the motor system; to endocrinology for risk assessment of insulin resistance; to rehabilitation therapy medicine for stimulation and monitoring of the motor system and to genetics for genetic counseling. The progressive degeneration of the motor system has disabled them to carry out basic activities, making them dependent.

\section{DISCUSSION}

Four cases were females out of the seven cases presented. More cases were reported from Huancayo, city of the Andes, where the commitment of AT between siblings was identified. Five cases were diagnosed after 3 years of started ataxia or telangiectasia. One case has total dependence and two cases died. The most frequent reason for consultation were recurrent lung infection, followed by postural instability. All had delayed psychomotor development, recurrent respiratory, and digestive infections. The most frequent laboratory results were lymphopenia, neutropenia, IgA deficiency, and elevated alpha-fetoprotein. 
All cases follow the classic model of AT motor disorders, consisting of postural instability and ataxic gait evidenced in the first 2 years of life. However, one of the cases has a later onset of symptoms, in the fifth year of life, which could be the objective of study to clarify the discrepancy. In one case, the onset of ataxia was after the appearance of ocular telangiectasias, a characteristic not reported in other series of cases reviewed.

In our case series affected siblings are generally observed in the same family. Three cases have a brother with AT. We have 2 families from Junín with 1 brother with AT at the diagnosis of the index case. The causal variants of the disease can be homozygous or compound heterozygous $(1,4)$. In the cases studied, only variants in compound heterozygosity are described, which are related to the non-consanguinity of the parents.

Recurrent respiratory infections being the reason for consultation (6/7), despite presenting neurological clinical characteristics. This could be due to underestimation of neurological symptoms or difficulty in accessing the health system. Reasons for which it should be investigated in greater depth.

Four cases have partial dependence ( $\leq 10$ years) and one case has total dependence ( $>10$ years) consistent with the literature, which refers that patients with AT can progressively reach total dependence at 10 years (1). In addition, two cases died, one case, at age 12 due to pneumonia in 2018 and another case, at age 9 due to respiratory failure in 2019. We found some reports with findings similar to ours (9).

IgA and IgG values are normal in two and six of the patients respectively, values that should be de- creased according to the literature (1), however, we found some reports with findings similar to ours (8-10).

Genetic counseling is recommended for all patients with AT in order to provide genetic consultation, preparation of the heredogram, pre-test and post-test genetic counseling, personal and family risk calculation, and preparation of an individualized management plan that should include prevention measures for the cancer.

Patients have limitations in the control of neurodegeneration and cannot be exposed to ionizing radiation due to a susceptibility to developing neoplastic diseases (1). However, 5 patients were exposed to X-ray imaging studies to study their recurrent respiratory infections.

This study is the first AT case series that includes an experience of 8 years in Peru. The limitation is memory bias since the information provided by the mother may have been affected by not remembering precise data.

\section{CONCLUSIONS}

AT considerably deteriorates the quality and prognosis of life of patients. Therefore, in patients with a family history of AT it is important to identify the suggestive clinical features for an early diagnosis. In addition, health personnel should consider AT within the differential diagnoses in children with delayed psychomotor development and ocular telangiectasias, thus reaching an early diagnosis, adequate follow-up and avoiding radiation damage.

Conflict of interest: none declared Financial support: none declared

\section{REFERENCES}

1. Rothblum-Oviatt C, Wright J, Lefton-Greif MA, McGrath-Morrow SA, Crawford TO, Lederman HM. Ataxia telangiectasia: a review. Orphanet J Rare Dis. 2016;11(1):159.

2. $\mathrm{Li} \mathrm{J}$, Jiang $\mathrm{D}$. The role of epigenomics in the neurodegeneration of ataxia-telangiectasia. Epigenomics. 2015;7(2):137-41.

3. Nissenkorn A, Ben-Zeev B. Ataxia telangiectasia. Handb Clin Neurol. 2015;132:199-214.

4. van Os NJH, Roeleveld N, Weemaes CMR, Jongmans MCJ, Janssens GO, Taylor AMR, et al. Health risks for ataxiatelangiectasia mutated heterozygotes: a systematic review, meta-analysis and evidence-based guideline. Clin Genet. 2015;90(2):105-17.

5. Devaney R, Pasalodos S, Suri M, Bush A, Bhatt JM. Ataxia telangiectasia: presentation and diagnostic delay. Arch Dis Child. 2017; 102(4):328-30.
6. McKinnon PJ. ATM and the Molecular Pathogenesis of Ataxia Telangiectasia. Annu Rev Pathol Mech Dis. 2012;7(1):303-21.

7. Paull TT. Mechanisms of ATM Activation. Annu Rev Biochem. 2015;84(1):711-38.

8. Lazo Rivera E, Vizcarra P, Fernando L. Ataxia-Telangiectasia: Reporte de caso. Rev Neuro-Psiquiatr. 2014;77(4):283-7.

9. Pascual-Pascual SI, Pascual-Castroviejo I, Fontan G, López-Martín V. Ataxia-telangiectasia (A-T). Contribution with eighteen personal cases. Brain Dev. 1981;3(3):289-96.

10. Lockman JL, Iskander AJ. The critically ill patient with ataxia telangiectasia: A case series. Pediatr Crit Care Med. 2012;3(2). 